\title{
High Precision Distance Relaying Algorithm for Transmission Line Protection by Optimal Integral Approximation Technique
}

\author{
Non-member Kyung-Rae Cho (Seoul National University) \\ Member Jong-Keun Park (Seoul National University) \\ Non-member Jun-Hee Hong (Kyungwon University)
}

\begin{abstract}
This paper presents a new distance relaying algorithm for transmission line protection and the method of estimating integral coefficients in this algorithm. The presented algorithm is based on the differential equation that satisfies the relation between the voltage and the current of transmission line. This algorithm calculates impedance values by approximating the integral term of the integro-differential equation, which is obtained by taking integral to differential equation. Spectrums of voltage and current due to faults of transmission lines are obtained by Prony's analysis. To find optimal integral coefficients in a least-square-error sense in the frequency domain, each weighting function of voltage and current coefficients can be derived by considering these spectrums and analog filter together. These weighting functions give the optimal coefficients appropriate to transient voltage and current after faults. The simulation and the test result show that the proposed algorithm can be used successfully to protect transmission lines.
\end{abstract}

Key words : Distance relay, Integral approximation, Prony's analysis, Integral coefficients

\section{Introduction}

A distance relay for transmission line protection calculates the impedance at the fundamental frequency from sampled voltage and current and determines whether faults have occurred or not. Since faults in power system are substantially a transient phenomena, post-faults voltage and current include DC-offset and many harmonics besides the fundamental frequency component. Distortion of fundamental components by these harmonics disturbs relay operations which should be precise and fast. Up to the present, various algorithms to estimate impedance from these distorted signals have been proposed, for example, differential equation, least square curve fitting and orthogonal function, etc. ${ }^{(1) \sim(10)}$.

Among these algorithms the orthogonal function method doesn't need a high-order analog filter due to its robust characteristics against harmonics. However, this method has a weak point that it can't increase the operation speed even if the sampling interval is shorter. It's because that the length of sampled data for detecting faults must be, at least, a half of the period of fundamental component. The others use the high-order analog filter to extract fundamental components from faults signal but the large time-delay by the filter causes the increase of operation time. In consequence, it is clear that to satisfy both high accuracy and high speed under the distortion of fault signals, distance relaying algorithm must not need a high-order analog filter and a lot of sampled data.

The differential equation method is one of the distance relaying algorithms that don't require many sampled data to detect faults. This method uses the differential equation of Eq. (1) and has robust characteristics against DC-offset due to its property.

$$
\nu=R i+L \frac{d i}{d t}
$$

To obtain line parameter values from Eq. (1), the differential term of the right side has to be estimated from sampled data. Ohura approximated this term in consideration of fundamental components and some harmonics nearby fundamental frequency ${ }^{(4)}$. On the other hand, the integral approximation method obtains impedance values by estimating the integral term of the integro-differential equation of Eq. (2) driven by taking integral to Eq. $(1)^{(3)(10)}$. The integral approximation method has more computational burden than the differential equation method but it has the advantage of robustness against harmonics ${ }^{(11)}$. 


$$
\int_{t_{0}}^{t_{1}} \nu(t) d t=R \int_{t_{0}}^{t_{1}} i(t) d t+L\left\{i\left(t_{1}\right)-i\left(t_{0}\right)\right\}
$$

In the beginning of digital relay, the trapezoidal rule was used to approximate the integral term but the approximation by this numerical method is inaccurate even in the signal composed of only the fundamental frequency component. To improve the accuracy of integral approximation, Kudo introduced midpoint interpolation from sampled values before applying the trapezoidal rule ${ }^{(3)}$. This integral approximation technique has many limitations of improving the accuracy of impedance calculation because of its inaccuracy of integral approximation in fundamental frequency.

This paper proposes a new algorithm which is based on the integro-differential equation and the numerical integral approximation technique. The proposed algorithm can calculate precisely the impedance of transmission lines from heavily distorted fault signals. To obtain very precise spectrums of fault signals. Prony's analysis is used and weighting functions are derived by considering these spectrums and the characteristics of an analog filter together. These weighting functions give the integral coefficients in a least-square-error sense in the frequency domain. The simulation and the test result show that the proposed algorithm can detect faults succssfully.

\section{Proposed integral approximation algorithm}

In distance relaying algorithms based on the differential equation, a transmission line can be modeled as an equivalent circuit of Fig. 1.

In this circuit the relation between the voltage and the current satisfies Eq. (1) and integrating this equation with the sampled data gives an integro-differential equation, that is

$$
\begin{aligned}
\int_{t_{0}}^{t_{0}+(M-1) T} \nu(t) d t= & R \int_{t_{0}}^{t_{0}+(M-1) T} i(t) d t \\
& +L\left[i\left\{t_{0}+(M-1) T\right\}-i\left(t_{0}\right)\right]
\end{aligned}
$$

where, $T$ : sample interval

$M$ : the number of sampled data to be used in integral approximations

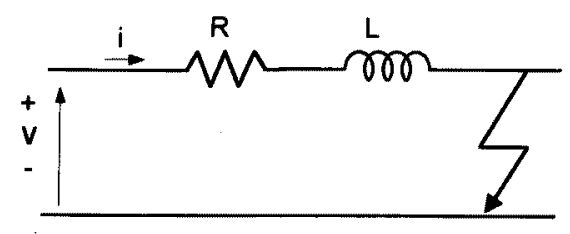

Fig. 1. Equivalent circuit of faulted transmission line.
Then the impldance values can be obtained from two simultaneous equations of Eq. (3)

$$
\begin{aligned}
& {\left[\begin{array}{l}
R \\
L
\end{array}\right]} \\
& =\left[\begin{array}{ll}
\int_{t_{0}}^{t_{0}+(M-1) T} i(t) d t \quad i\left\{t_{0}+(M-1) T\right\}-i\left(t_{0}\right) \\
\int_{t_{0}}^{t_{0}+(M-2) T} i(t) d t & i\left\{t_{0}+(M-2) T\right\}-i\left(t_{0}-T\right)
\end{array}\right]^{-1} \\
& \\
& \times\left[\begin{array}{l}
\int_{t_{0}}^{t_{0}+(M-1) T} \nu(t) d t \\
\int_{t_{0}-T}^{t_{0}+(M-2) T} \nu(t) d t
\end{array}\right] \ldots \ldots \ldots \ldots \ldots \ldots \ldots \ldots(4)
\end{aligned}
$$

To get the values of $R$ and $L$ in Eq. (4), a numerical technique that can approximate the integral term of the right side is necessary. The following numerical integral technique is selected ${ }^{(10)}$.

$$
\begin{aligned}
\int_{t_{0}}^{t_{0}+(M-1) T} \nu(t) d t= & \sum_{k=1}^{N} C_{k}\left[\nu\left\{t_{0}+(k-1) T\right\}\right. \\
& \left.+\nu\left\{t_{0}+(M-k) T\right\}\right]
\end{aligned}
$$

where $C_{k}$ : integral coefficients

$N$ : the number of integral coefficients

As shown in Eq. ( 5 ), the proposed integral approximation method has a symmetrical structure like the Trapezoidal rule. Therefore, it has a linear phase characteritics in frequency domain ${ }^{(1)}$.

Since the integral coefficients give a great effect on the performance of integral approximation, it is critically important to determine proper integral coefficients. Choosing $N$ frequencies, in which the integral approximation errors are zero, we get the following equation to find $C_{k}$.

$$
\begin{gathered}
{\left[\begin{array}{c}
C_{1} \\
C_{1} \\
\vdots \\
C_{N}
\end{array}\right]=\left[\begin{array}{cccc}
A_{11} & A_{12} & \cdots & A_{1 N} \\
A_{21} & A_{22} & \cdots & A_{2 N} \\
& \vdots & \vdots & \vdots \\
A_{N 1} & A_{N 2} & \cdots & A_{N N}
\end{array}\right]^{-1}\left[\begin{array}{c}
B_{1} \\
B_{2} \\
\vdots \\
B_{N}
\end{array}\right]} \\
\text { where } A_{i j}=\cos \frac{\omega_{1}(M+1-2 j) T}{2} \\
B_{i}=\frac{1}{\omega_{i}} \sin \frac{\omega_{i}(M-1) T}{2}
\end{gathered}
$$

In Eq. ( 6 ) the necessary condition to get coefficients uniquely is $M \geq 2 N-1$ due to its structure of Eq. (6). It is desirable that the value of $M$ should be the smallest value $2 N-1$ since the operation time of relay is related directly to the number of sampled data in the approximation of the integral term.

In Eq. (6) $N$ frequencies to determine integral coefficients may be the fundamental frequency, $0 \mathrm{~Hz}$ (that is DC-offset), the second harmonic and so on. This selection of frequencies to find the integral coefficients can't refiect the characteristics of fault signals effectively and the efficient determination of 
integral coefficients can be achieved through the accurate analysis of fault signals in the frequency domain.

\section{Determination of integral coefficients}

\subsection{Spectrums of fault signal by Prony's analysis}

Prony's analysis is a well-known technique of analyz ing signals to determine modal, damking, phase and magnitude information contained in the signal ${ }^{(12) \sim(14)}$. It is an extenison of Fourier analysis in that damping information as well as frequency information is otained. Prony's analysis gives an optimal fit in the leastsquare-error sense to output signal $y(t)$ in the form

$$
y(t)=\sum_{k=1}^{P_{N}} B_{k} \exp \left(\lambda_{k} t\right)
$$

where $P_{N}$ : the number of mode in signal

The $s$-domain representation of the signal can be directly obtained from Eq. (7) and letting $s=j \omega$ gives the frequency characteristics of the signal $y(t)$ as shown in Eq. (8).

$$
Y(s)=\sum_{k=1}^{F_{N}} \frac{B_{k}}{s-\lambda_{k}} \text { or } \quad Y(\omega)=\sum_{k=1}^{P_{N}} \frac{B_{k}}{j \omega-\lambda_{k}}
$$

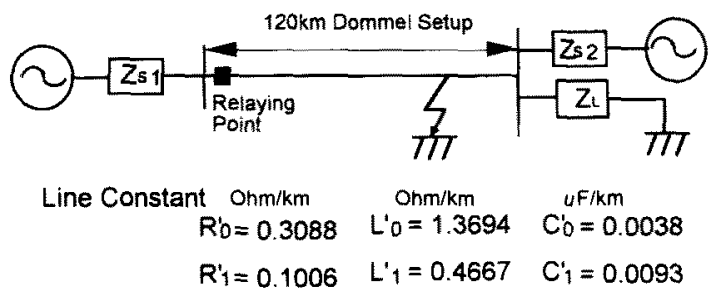

Fig. 2. Fault model for Prony's analysis.

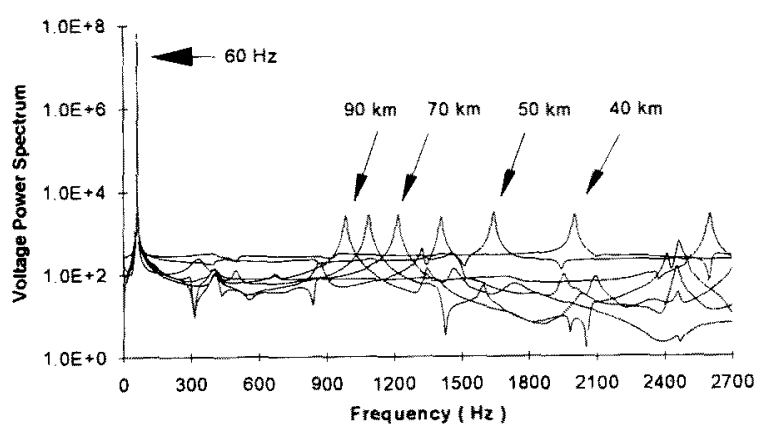

(a) Voltage spectrum

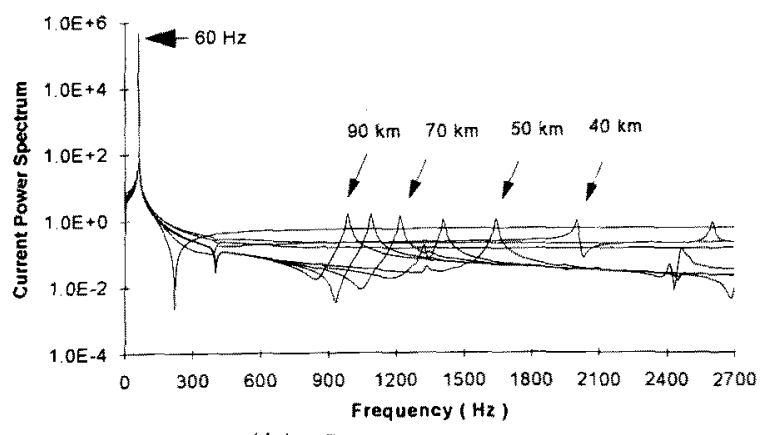

(b) Current spectrum

Fig. 3. Spectrums at a voltage angle of $90^{\circ}$.
The fault data to be analyzed by Prony's analysis are obtained from EMTP (ElectroMagnetic Transient Program) and the line model is the Dommel setup.

Fig. 3 shows the spectrums of voltage and current for each fault location from $10 \mathrm{~km}$ to $90 \mathrm{~km}$ after the occur. rences of $A$-phase to earth faults at a voltage angle of $90^{\circ}$.

The oscillation frequency which exists dominantly in both the voltage and the current spectrum of Fig. 3 is known to be caused by reactant and capacitance components of transmission lines. If the fault location from the relaying point becomes more distant, the dominant oscillation frequencies get nearer to the fundamental frequency.

Fig. 4 shows the spectrums of the voltage and the current for each fault angle from 0 to $90^{\circ}$ after the occurrences of $A$-phase to earth faults at a fault distance of $90 \mathrm{~km}$.

In this figure it is shown that both voltage and current spectrums have similar aspects for each fault angles but the magnitudes of the spectrums are increased as the fault angle varies from 0 to $90^{\circ}$.

Through Prony's analysis of fault signals we can also confirm the fact that voltage spectrums in the range of frequency over the fundamental frequency are greater in magnitude than those under the fundamental frequency but current spectrums show the contrary result to voltage spectrums. This indicates that the integral

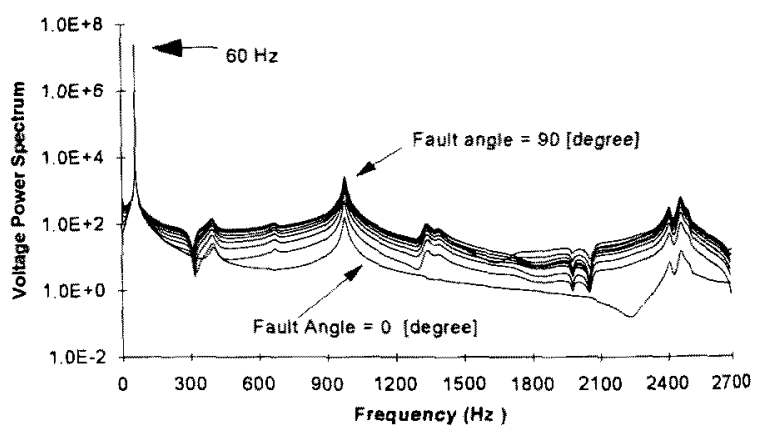

(a) Voltage spectrum

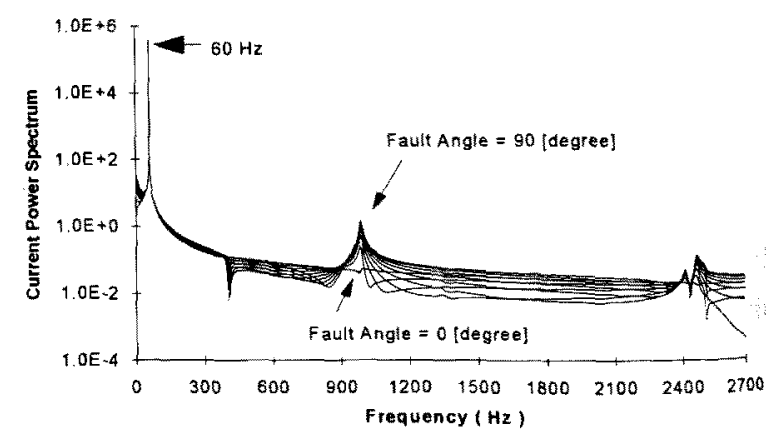

(b) Current spectrum

Fig. 4. Spectrums at a fault location of $90 \mathrm{~km}$. 
coefficients of voltage should be different from those of current.

\subsection{Optimal depermination of integral coefficients}

Define the sinusoidal function $h_{f}(t)$ for a certain frequency $f$ as shown below,

$$
\begin{aligned}
& h_{f}(t)=H_{f} \sin \left(2 \pi f t+\theta_{f}\right) \cdots \cdots \cdots \cdots \cdots \cdots \cdots \cdots \cdots(9) \\
& \text { where } H_{f}: \text { the magnitude of sinusoid with fre- }
\end{aligned}
$$
quency $f$

Then the analytic integral value for a certain time interval $\left\lfloor t_{0}, t_{0}+(M-1) T\right\rfloor$ is

$$
\begin{aligned}
g_{f} & =\int_{t_{0}}^{t_{0}+(M-1) T} h_{f}(t) d t \\
& =\frac{H_{f}}{\pi f} \sin \phi_{f} \sin \{\pi f(M-1) T\}
\end{aligned}
$$

$$
\text { where } \varphi_{f}=2 \pi f t_{0}+\theta_{f}+\pi f(M-1) T
$$

And the approximation value of $h_{f}(t)$ for the same interval in Eq. (10), by the help of Eq. (5), is

$$
\begin{aligned}
\hat{g}_{f} & =\sum_{k=1}^{N} C_{k}\left[h_{h}\left\{t_{0}+(k-1) T\right\}+h_{h}\left\{t_{0}+(M-k) T\right\}\right] \\
& =2 H_{f} \sin \varphi_{f} \sum_{k=1}^{N} C_{k} \cos \{\pi f(M-1-2 k) T\} .
\end{aligned}
$$

In both Eq. (10) and Eq. (11), $\sin \varphi_{f}$ is determined by $\theta_{f}$, the initial phase at the beginning of the faults. Regarding the initial phase as a probability variable distributed uniformly from 0 to 2 , the error between the analytic integral value and the approximation value are defined as

$$
\begin{aligned}
E(f)^{2}= & \frac{1}{2 \pi} \int_{0}^{2 n}\left(g_{f}-\hat{g}_{f}\right)^{2} d \varphi_{f} \\
= & \frac{H_{f}^{2}}{2}\left[\frac{1}{\pi f} \sin \{\pi f(M-1) T\}\right. \\
& \left.-2 \sum_{k=1}^{N} C_{k} \cos \{\pi f(M-1-2 k)\}\right]^{2}
\end{aligned}
$$

where $H_{f}=M_{f} w_{l p}(f)$

$M_{f}$ : magnitude characteristics of analog filter

$w_{l p}(f)$ : spectrum obtained through Prony's analysis for fault at location $l$, voltage angle $p$ degree

Total integral approximation error, by integral coefficients $C_{k}$ 's, should be obtained by considering the fault location, the fault angle and the cutoff frequency of an analog filter. This total error can be written as

$$
\begin{aligned}
E_{\text {total }}^{2}= & \sum_{l=0}^{D_{l}} \sum_{p=0}^{g 0^{\circ}} \sum_{f=0}^{f_{c}} E_{l p}(f)^{2} \\
= & \sum_{f=0}^{f_{c}} W(f)^{2}\left[\frac{1}{\pi f} \sin \{\pi f(M-1) T\}\right. \\
& \left.-2 \sum_{k=1}^{N} C_{k} \cos \{\pi f(M-1-2 k) T\}\right]^{2}
\end{aligned}
$$

where $W(f)^{2}=M(f)^{2} \sum_{l=0}^{D_{l}} \sum_{p=0}^{90^{\circ}} w_{l p}(f)^{2}$
$D_{l}$ : distance of transmission line to be protected

In Eq. (13), $W(f)$ represents the characteristics of transmission lines considering the magnitude characteristics of an analog filter and the frequency characteristics of fault signals together, and it is a weighting function to find the integral coefficients.

The integral coefficients should minimize the squared total error of Eq. (13) and these coefficients make partial derivatives for each coefficients zero. That is

$$
\begin{aligned}
\frac{\partial E_{\text {total }}^{2}}{\partial C_{j}}= & -4 \sum_{f=0}^{f_{c}} W(f)^{2}\left[\frac{1}{\pi f} \sin \{\pi f(M-1) T\}\right. \\
& \left.-2 \sum_{k=1}^{N} C_{k} \cos \{\pi f(M-1-2 k) T\}\right] \\
& \times \cos \{\pi f(M-1-2 j) T\} \\
= & 0 \quad \text { for } j=1, \cdots, N
\end{aligned}
$$

Arranging this partial derivative equation in matrix form for every coefficient gives optimal coefficients to minimize the square integral approximation error.

$$
\begin{gathered}
{\left[\begin{array}{c}
C_{1} \\
C_{1} \\
\vdots \\
C_{N}
\end{array}\right]=\left[\begin{array}{cccc}
Z_{11} & Z_{12} & \cdots & Z_{1 N} \\
Z_{21} & Z_{22} & \cdots & Z_{2 N} \\
\vdots & \vdots & \\
Z_{N 1} & Z_{N 2} & \cdots & Z_{N N}
\end{array}\right]^{-1}\left[\begin{array}{c}
D_{1} \\
D_{2} \\
\vdots \\
D_{N}
\end{array}\right] \cdots \cdots \cdots \cdots \cdots \cdots(15)} \\
\text { where } Z_{y}=\sum_{f=0}^{f_{c}} W(f)^{2}\left[\frac{1}{\pi f} \cos \{\pi f(M-1-2 j) T\}\right. \\
\quad \times \cos \{\pi f(M-1-2 i) T\} \\
D_{i}=\frac{1}{2} \sum_{f=0}^{f c} 2 W(f)^{2} \frac{1}{\pi f} \sin \{\pi f(M-1) T\} \\
\quad \times \cos \{\pi f(M-1-2 i) T\}
\end{gathered}
$$

The sampling speed $(1 / T)$ and the number of integral coefficients are to be determined. The sampling speed used commonly is $720 \mathrm{~Hz}$ in the $60 \mathrm{~Hz}$ system. The sampling speed of $1,440 \mathrm{~Hz}$ is expected to be used sooner or later. The number of coefficients influences the accuracy and the speed of relay operation. If this number is too small, the operation speed of the relay becomes fast but its accuracy is not guaranteed. On the other hand, a large number of coefficients give more accurate impedance values but the operation speed gets down. Therefore the number of coefficients should be determined to keep the balance between the operation speed and the accuracy of the relay. As a result of study, it is appropriate that the number of integral coefficients be 2 in $720 \mathrm{~Hz}$ sampling speed and 3 in 1,440 $\mathrm{Hz}$.

\section{Simulation and test result}

\subsection{Coefficients}

The characteristics of an analog filter is needed to find the integral coefficients. In general, an analog filter is 
used to prevent the aliasing error caused by A/D conversion and it must remove the frequency components of the stop band effectively without a large time delay. Since the proposed algorithm in this paper uses various ferquency components as well as the fundamental component, a second-order Butterworth filter which has 0.96 magnitude characteristics and 0.79 radian phase characteristics at $60 \mathrm{~Hz}$ is used in the relay and its characteristics is plotted in Fig. 5.

The major requirements to determine the integral coefficients are as follow.

(1) The time to detect faults is shorter than a half cycle of the fundamental frequency.

(2) The maximum error of the estimated fault distance is no more than $5 \%$.

(3) A/D converter has 12 bit resolution and 2 LSB error.

To satisfy the requirements of ( 1 ), the minimum sampling frequency is 24 samples/cycle since the anlog filter causes the time delay as mentioned above and the size of data window to be used to estimate line parameters needs 4 samples at least. As a result of simulation and test, the number of integral coefficients should be equal or greater than 3 to satisfy the requirement of (2) under the condition of the requirement (3). Selecting 3 as the number of integral coefficients, sample data of 5 or 6 may be needed to calculate the integral term. It is more desirable to select the odd number, that

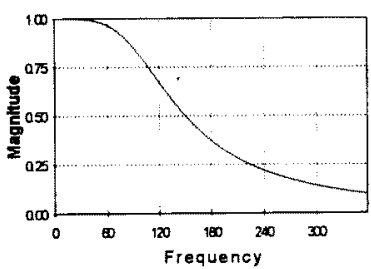

(a) magnitude

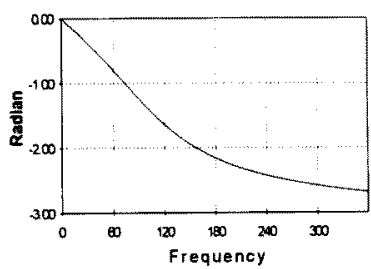

(b) phase
Fig. 5. Characteristics of 2nd-order Butterworth filter.

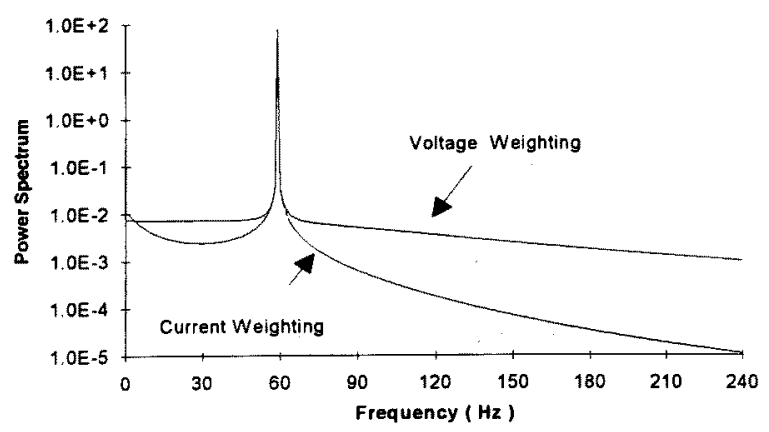

Fig. 6. Weighting function of voltage and current coefficients. is 5 , as the number of data to be used in integral approximation since it is smaller than 6 and has a merit that odd number makes it simple to sample at $1,440 \mathrm{~Hz}$ and to calculate line parameter every $1 / 720 \mathrm{~s}$. All specifications mentioned above to find the integral coefficients are summarized in Table 1.

Fig. 6 shows the voltage and the current weighting functions, respectively. In Fig. 7 the approximation errors by the integral coefficients according to Table 1 is plotted while the values of the weighting functions are all 1 for all frequencies to reveal the nature of the integral coefficients.

From Fig. 7, it is found that the integral coefficients of voltage make the approximation error zero at $60 \mathrm{~Hz}$, $117 \mathrm{~Hz}$ and $213 \mathrm{~Hz}$ and in case of the current, the approximation errors are zero at $5 \mathrm{~Hz}, 32 \mathrm{~Hz}$ and $60 \mathrm{~Hz}$. The integral coefficients of the current have small approximation error at the frequencies under the fundamental frequency.

Those of the voltage have small integral approximation error up to 3.5 times the fundamental frequency. The approximation errors of both coefficients at $60 \mathrm{~Hz}$ are zero as predicted in the weighting function. In addition it could be found that if the cutoff frequency of the analog filter is higher, the frequency in which the approximation error remains almost zero is also higher.

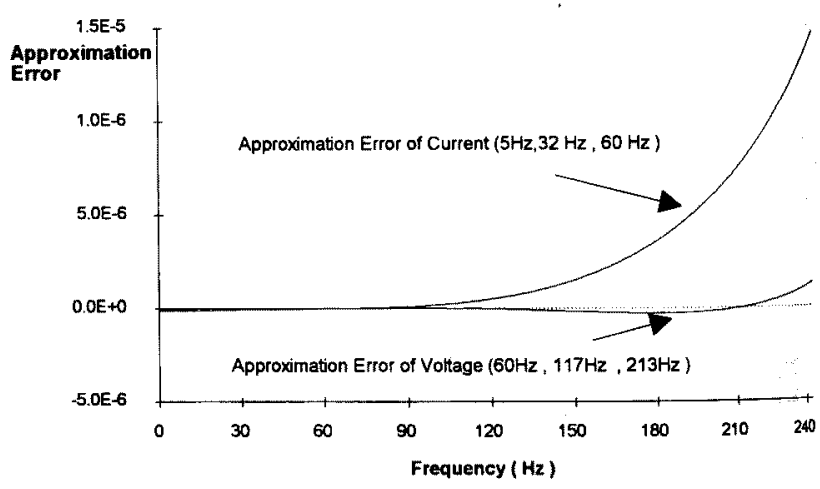

Fig. 7. Approximation errors by current and voltage coefficients.

Table 1. Specification to find the integral coefficients.

\begin{tabular}{c|c}
\hline Item & Specification \\
\hline Fundamental Frequency & $60 \mathrm{~Hz}$ \\
Sampling Speed & $1,440 \mathrm{~Hz}$ \\
& $(24$ samples/cycle $)$ \\
Number of Coefficients & 3 \\
Number of data used in & 5 \\
integral approximation & \\
Analog Filter & 2nd.order Butterworth \\
& with $f_{c}=360 \mathrm{~Hz}$ \\
\hline
\end{tabular}




\subsection{Comparison with interpolated integral method}

The interpolated integral method ${ }^{(3)}$ used 4 sample data to approximate integral term at the sampling frequency of $720 \mathrm{~Hz}$ as Eq. (16).

$$
\begin{aligned}
& S I_{k}=\left(i_{k}+i_{k-1 / 2}\right) \frac{T}{4}+\left(i_{k-1 / 2}+i_{k+1}\right) \frac{T}{4} \\
& i_{k-1 / 2}=i_{k-1}+k_{1} i_{k}^{\prime}+k_{2} i_{k-1}^{\prime} \\
& \text { where } i_{k}^{\prime}=\frac{i_{k+1}-i_{k-1}}{2 T}
\end{aligned}
$$

Eq. (16) can be rewritten in terms of sampled data.

$$
\begin{aligned}
& S I_{k}=\frac{T}{4}\left[\frac{k_{1}}{T} i_{k+1}+\left(1+\frac{k_{2}}{T}\right) i_{k}+\left(2-\frac{k_{1}}{T}\right) i_{k-1}-\frac{k_{2}}{T} i_{k-2}\right] \\
& =A i_{k+1}+B i_{k}+C i_{k-1}+D i_{k-2}
\end{aligned}
$$

As shown in Eq. (17), the interpolated integral method does not have a symmetric structure since it can not satisfy $A=B$ and $C=D$ simultaneously. Thus, the integral approximation error depends on the initial phase of the sampled data.

To compare the proposed method with the interpolated integral method, we apply 4 data points $-720 \mathrm{~Hz}$ sampling frequency scheme to our method. Fig. 8 shows the approximation error curves of the interpolated integral method for 3 initial phase of $0.5,1.0,1.5$ radian respectively and that of proposed integral approximation method. These error curves were obtained using the same method used in the Section 4.1 coefficients. It is clearly seen that the error curves of the interpolated

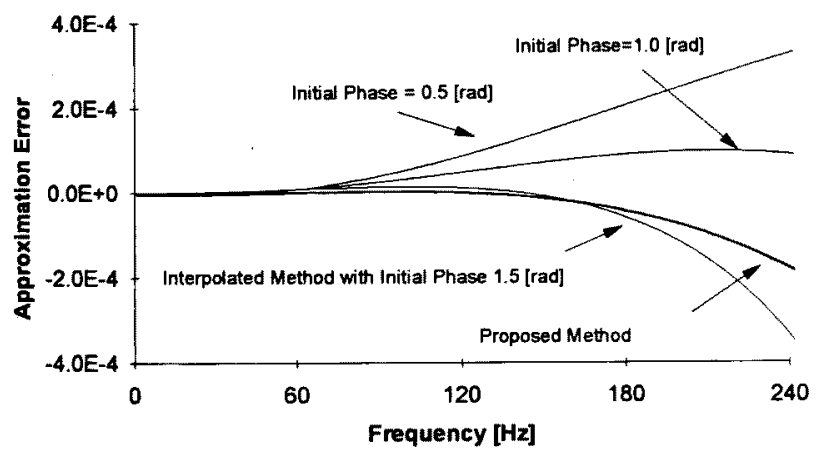

Fig. 8. Integral approximation error by the interpolated integral method compared with the proposed method.

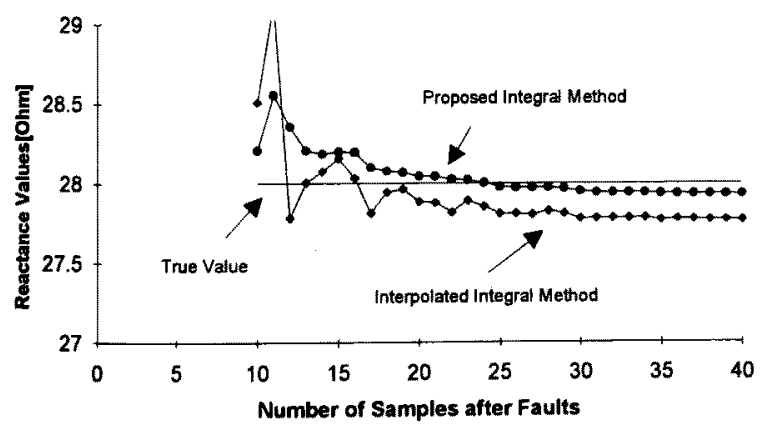

Fig. 9. Reactance values by two integral methods. integral method change according to the initial phase. On the other hand the approximation error of the proposed integral method does not be affected by the initial phase. This is mainly due to its symmetric structure as mentioned above. It is also found in Fig. 8 that the proposed method has very small errors up to about $180 \mathrm{~Hz}$ while the error of the interpolated integral method starts to grow at about $60 \mathrm{~Hz}$. The large errors above $180 \mathrm{~Hz}$ can be reduced by an analog filter as used in many other distance algorithms.

Fig. 9 shows an example of the estimated rectance by the two integral methods. The estimated resistance is not shown here for the two methods have almost the same result. In Fig. 9, however, it is clear that the reactance result of the proposed integral method has smaller ripple and better accuracy than that of interpolated integral method.

\subsection{Simulation}

The fault location can be determined using the positive reactance value obtained from voltage and current and may be inaccurate due to much harmonics at faults which occur at a voltage angle nearby $90^{\circ}$. Fig. 10 shows the calculated positive reactance value for each location of earth to phase faults at a fault angle of $90^{\circ}$. As shown in Fig. 10, the reactance values have little fluctuations though the faults occurred at a voltage angle of $90^{\circ}$.

Fig. 11 shows the mean values of absolute error of the converged resistance and reactance values for each fault location and each fault angle.

Fig. 11 also indicates that the reactance values are more accurate than the resistance value. It's because the compensation method of the zero-sequence current to obtain the positive impedance value from each phase voltage and current focuses on the reactance values.

The time, in which the impedance after faults converges to the value within $5 \%$ of the true value, ranges from minimum 8 sample intervals $(6 \mathrm{~ms})$ to maximum 12 sample intervals ( $9 \mathrm{~ms}$ ) as indicated in the impedance locus of Fig. 12.

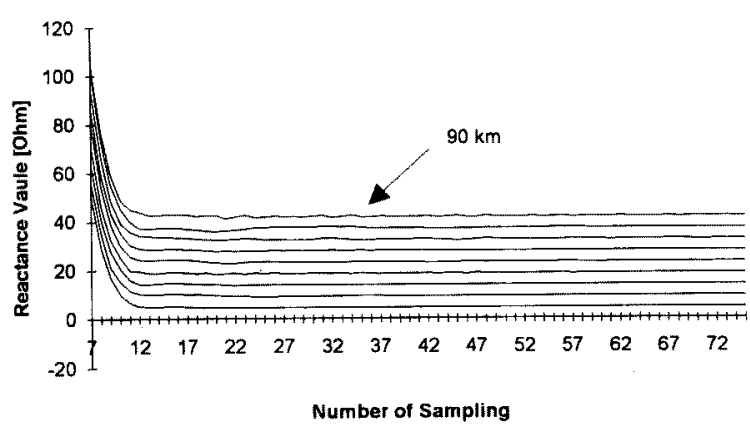

Fig. 10. Reactance values at a fault angle of $90^{\circ}$. 


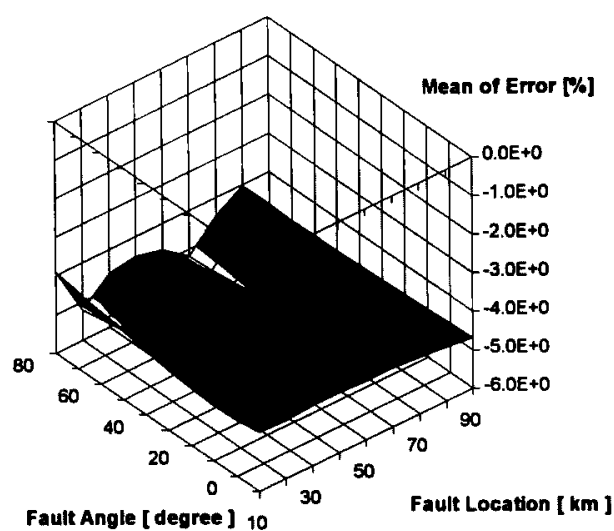

(a) Error of resistance

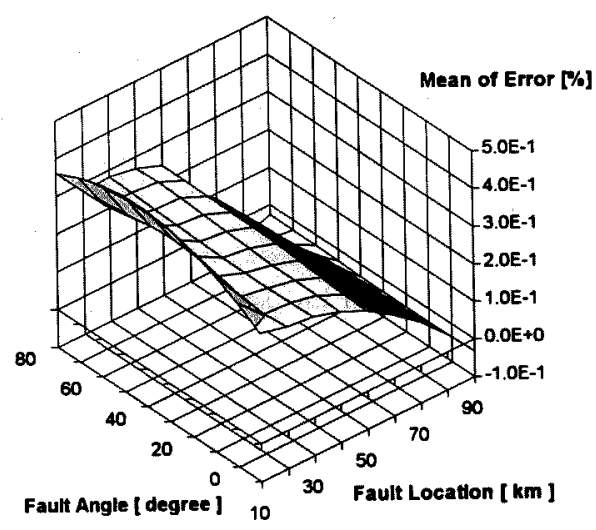

(b) Error of reactance

Fig. 11. Mean value of absolute error of the converged impedance value.

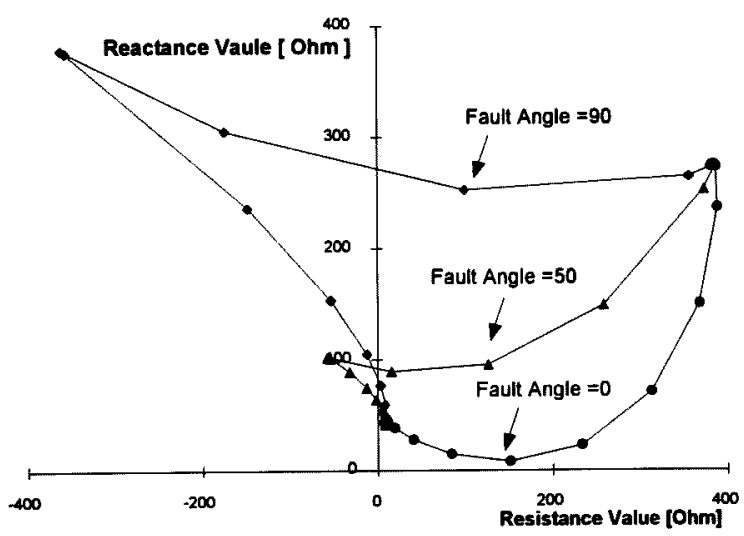

Fig. 12. Impedance locus after faults.

\subsection{Test result}

The proposed algorithm is programmed in a prototype relay that was developed in cooperation with KEPCO (Korea Electric Power Corporation) in 1992 and it has a 12 bit A/D converter, the Intel 80286 microprocessor and a DSP to resolve the computational burden. A relay tester with an output capability of $30 \mathrm{~A}$ maximum current, $100 \mathrm{~V}$ maximum voltage and various frequencies as well as $60 \mathrm{~Hz}$ was employed to test the proposed relaying algorithm. The configuration of the

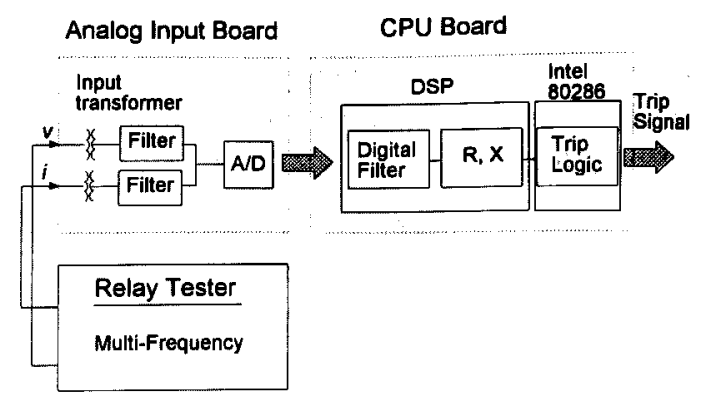

Fig. 13. Test configuration of $\mathrm{H} / \mathrm{W}$ and $\mathrm{S} / \mathrm{W}$ of relay.

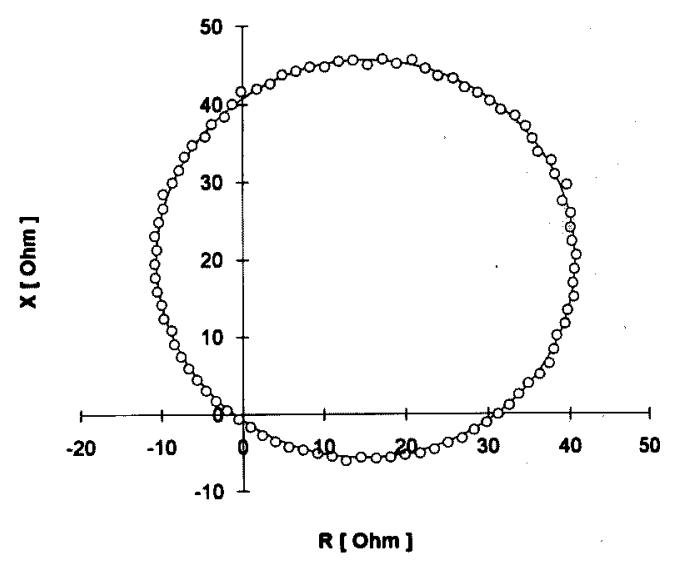

Fig. 14. Employed Mho-type trip zone and trip result.

hardware and the software to test the relay is plotted in Fig. 13.

The sampled data after A/D conversion have about 2 LSB error and the digital filter shown in Fig. 13 reduces this error of sampled data and it compensates the difference of phase delay between PT and CT.

The trip zone adopted in trip logic is Mho-type in which the trip radius enlarges $1 \Omega$ to include the origin of $R-X$ plane to increase sensitivity for close-up faults from bus bar. The inside of the solid-line circle shown in Fig. 14 indicates the trip zone that is installed at the relay and the many small circles nearby the solid-line circle represent the tested boundary points of impedance values, in which the relay operates perfectly within 50 ms after faults when the RMS value of the fault current is even equal to that of the steady-state current, which is the worst case in practice.

The maximum deviation of the tested boundary points from the trip zone at $100 \%$ current scale is $4.7 \%$ and the average value is $0.2 \%$. As the RMS value of the fault current increases, the tested boundary points get closer to the trip zone. The deviation curves for each ratio $\left|I_{f}\right| /\left|I_{\text {steady }}\right|$ are plotted in Fig. 15.

It can be easily found that as the current ratio increases, the relay operation becomes more accurate 


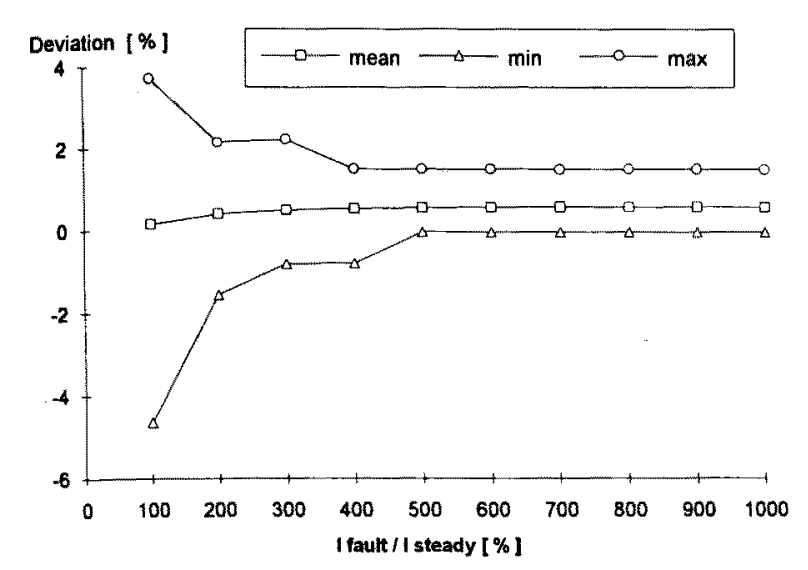

Fig. 15. The deviation from trip zone vs. $\left|I_{\text {taut }}\right| /\left|I_{\text {steady }}\right|$.

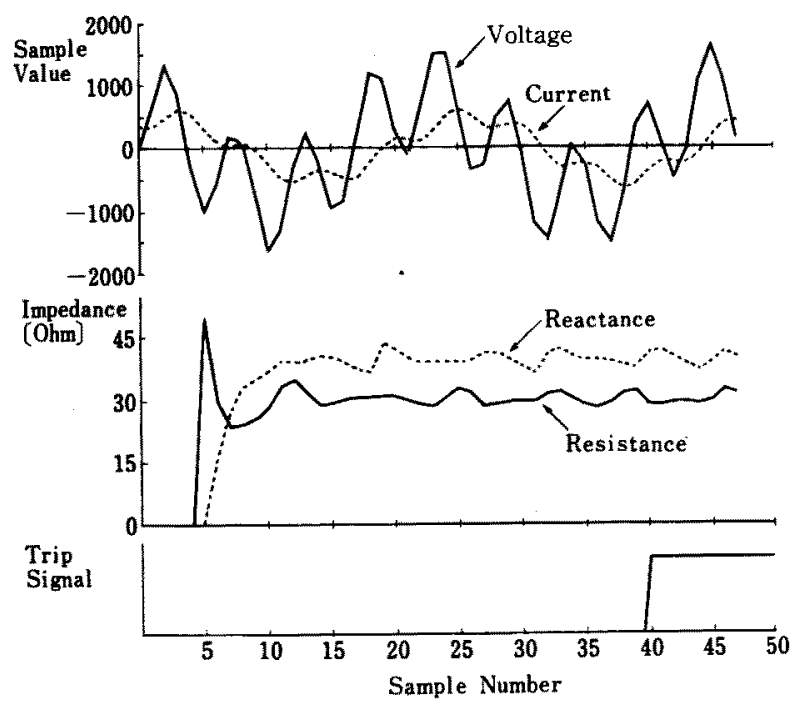

Fig. 16. Impedance calculation and trip signal for signals composed of other frequency components.

until the current ratio reaches $500 \%$. However, above $500 \%$ the accuracy remains the same. Furthermore the average value of the deviation is almost the same for all ranges of current ratio, which means that it is rarely affected by the magnitude of current.

Fig. 16 shows the results of impedance calculation and the trip signal of trip logic when a harmonic current which is $30 \%$ of the fundamental current in magnitude is added to the fault current.

The test input voltage has a larger magnitude of harmonic component compared with that of the harmonic current according to the differential equation of Eq. (1). In this case it is clear that the calculated impedance has some fluctuation and the operation of relay also has some delay. On the whole, it takes about 40 sampling intervals $(27 \mathrm{~ms}$ ) to decide the trip of the relay. The real output delay of the D 1/O (Digital Input and Output) board doesn't exceed $50 \mathrm{~ms}$.

\section{Conclusion}

A high precision distance relaying algorithm based on the differential equation using integral approximation technique is presented in this paper. Spectrums of the voltage and the current at $A$-phase to earth faults in transmission lines can be obtained by Prony's analysis. Each weighting function of the voltage and the current coefficients can be derived by considering these spectrums and the magnitude characteristics of analog filter together. These weighting functions give the integral coefficients appropriate to approximate the integral term from the transient voltage and current after faults. The simulation and test results show that the proposed distance relaying algorithm can be used successfully to protect transmission lines.

(Manuscript received Apr. 13, '95, revised Aug. 11, '95)

\section{References}

(1) B. Jeysura \& W. J. Smolinski: "Identification of a Best algorithm for Digital Distance Protection of Transmission Lines", IEEE Trans. Power Apparatus Syst., PAS-102, No. 10, 3358 $\sim 3369$ (1983)

(2) A. M. Ranibar \& B. J. Cory: "An Improved Method for the Digital Protection of High Voltage Transmission Lines", ibid., PAS-94, 544 550 (1975)

(3) H. Kudo, H. Sasaki, K. Seo, M. Takahash, K. Yoshida \& T. Maeda: "Implementation of a Digital Distance Relay Using an Interpolated Integral Solution of a Differential Equation", IEEE Trans. Power Delivery, 3, No. 4, 1475 1484 (1988)

(4) Y. Ohura, T. Matsuda, M. Suzuki, M. Yamaura, Y. Kurosawa \& T. Yokoyama: "Digital Distance Relay with Improved Characteristics Against Distorted Transient Waveforms", ibid., 4, No. 4, 2025 2031 (1988)

(5) B. J. Mann \& I. F. Morrison: "Relaying a Three Phase Transmission Line with Digital Computer", IEEE Trans. Power Apparatus Syst., PAS-90, No. 2, 742 750 (1971)

(6) IEEE Committee Report: "EHV Protection Problems", ibid., PAS-100, No. 5 (1981)

(7) S. M. Merry \& E. R. Taylor: "Overvoltages and Harmonics on EHV Systems", ibid., PAS-91, No.6 (1972)

(8) H. Kudo, et al.: "Development of New Distance Relays to Cope with Natural Frequency Transients in UHV/EHV Transmission Systems", ibid., PAS-104, No. 12 (1985)

(9) M. Okamura, et al.: "Development of New Relays with Significantly Improved Performance against Badly Distorted Transient Waveforms", ibid, PAS-99, No. 4 (1980)

(10) K. R. Cho, Byung-Tae Jung, Jong-Keun Park: "A New Distance Relaying Algorithm with Integral Approximation Technique", IEE of Japan Power \& Energy '94, pp. 167 -171

(11) R. L. Burden \& J. D. Fairs: "Numerical Analysis", PWSKENT, pp. 145 214 (1989)

(12) Jun-Hee Hong, Jong-Keun Park: "Time-Domain Reduction Method Using Prony, Analysis for Electromagnetic Transients Study", '95 IEEE Winter Meeting

(13) Tufts \& R. Kumareasan: "Singular Value Decomposition and Improved Frequency Estimation Using Linear Prediction", IEEE Trans. Acoustics, Speech \& Signal Processing, ASSP-30, No. 4, 671 675 (1982)

(14) Rao \& K. S. Arun: "Model Based Processing of Signal: A State Space Approach", Proc. IEEE, 80, No. 2 (1992) 
Kyung-Rae Cho (Non-member) He received the B. S. and $\left(\frac{1}{a}=1\right.$ M.S. degrees in Electrical Engineering from Seoul National University, Korea in 1990 and 1992, respectively. His areas of interest are power system protection and the transient analysis of power system. He is a member of IEEE and the Korean Institute of Electrical Engineers.

Jong-Keun Park (Member) He received the B. S. degree in -1
$z$
$z$ Electrical Engineering from Seoul National University, Korea in 1973 and the M. S. and $\mathrm{Ph}$. $\mathrm{D}$ degrees in Electrical Engineering from the University of Tokyo, Japan in 1979 and 1982, respectively. He joined the Faculty of the Department of Electrical Engineering, Seoul National University in $1983 . \quad \mathrm{He}$ is currently a Professor and the Chairman of the Department of Electrical Engineering, Seoul National University. In 1992, he was a visiting scholar at Massachusetts Institute of Technology. His fields of interest are in power system protection, artificial intelli. gence and its applications to power systems. He has been a member of International Advisory Committee of Expert System Applications to Power Systems since 1988. He is a member of the Korean Institute of Electrical Engineers, IEE of Japan and IEEE.

Jun-Hee Hong (Non-member) He received the B. S., M. S

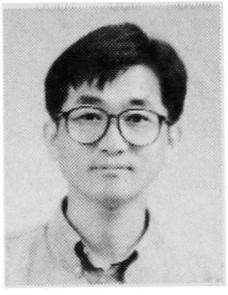
and $\mathrm{Ph}$. D. degrees in Electrical Engineering from Seoul National University, Korea in 1987, 1989, 1995 respectively. He is currently a Lecuturer of the Department of Electrical Engineering of Kyungwon University. His areas of interest are the equivalent reduction of power system and the transient analysis of power system. He is a member of IEEE and the Korean Institute of Electrical Engineers. 